\title{
The association between early-onset schizophrenia with employment, income, education, and cohabitation status: nationwide study with 35 years of follow-up
}

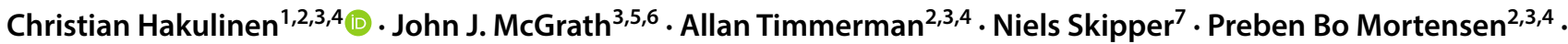 \\ Carsten Bøcker Pedersen ${ }^{2,3,4}$. Esben Agerbo ${ }^{2,3,4}$
}

Received: 20 February 2019 / Accepted: 20 August 2019 / Published online: 27 August 2019

(c) The Author(s) 2019

\begin{abstract}
Purpose Individuals with schizophrenia have been reported to have low employment rates. We examined the associations of schizophrenia with employment, income, and status of cohabitation from a work life course perspective.

Methods Nationwide cohort study including all individuals $(n=2,390,127)$ born in Denmark between 1955 and 1991 , who were alive at their 25th birthday. Diagnosis of schizophrenia (yes/no) between ages 15 and 25 was used as an exposure. Employment status, annual wage or self-employment earnings, level of education, and cohabitant status from the age of 25-61 (years 1980-2016) were used as outcomes.

Results Schizophrenia diagnosis between ages 15 and $25(n=9448)$ was associated with higher odds of not being employed (at the age of 30: OR 39.4, 95\% CI 36.5-42.6), having no secondary or higher education (7.4, 7.0-7.8), and living alone (7.6, 7.2-8.1). These odds ratios were two-to-three times lower and decreasing over time for those individuals who did not receive treatment in a psychiatric inpatient or outpatient clinic for schizophrenia after the age of 25. Between ages $25-61$, individuals with schizophrenia have cumulative earning of $\$ 224,000$, which is $14 \%$ of the amount that the individuals who have not been diagnosed with schizophrenia earn.

Conclusions Individuals with schizophrenia are at high risk of being outside the labour market and living alone throughout their entire life, resulting in an enormous societal loss in earnings. Individuals with less chronic course of schizophrenia had a gradual but substantial improvement throughout their work life.
\end{abstract}

Keywords Socioeconomic outcomes $\cdot$ Register-based study $\cdot$ Psychosis $\cdot$ Prospective study

\section{Introduction}

Electronic supplementary material The online version of this article (https://doi.org/10.1007/s00127-019-01756-0) contains supplementary material, which is available to authorized users.

Christian Hakulinen

christian.hakulinen@helsinki.fi

1 Department of Psychology and Logopedics, Faculty of Medicine, University of Helsinki, P.O. Box 21, 00014 Helsinki, Finland

2 iPSYCH, The Lundbeck Foundation Initiative for Integrative Psychiatric Research, Aarhus, Denmark

3 National Centre for Register-Based Research, Business and Social Sciences, Aarhus University, Aarhus V, Denmark
Schizophrenia is a poorly understood group of disorders, with reduced life expectancy from 10 to 20 years [1], affecting globally over 21 million individuals [2]. Schizophrenia is known to impact in all aspects of life; the first onset of

4 Centre for Integrated Register-Based Research, CIRRAU, Aarhus University, Aarhus, Denmark

5 Queensland Brain Institute, The University of Queensland, St Lucia, Australia

6 Queensland Centre for Mental Health Research, The Park Centre for Mental Health, Wacol, QLD, Australia

7 Department of Economics and Business Economics, Aarhus University, Aarhus, Denmark 
schizophrenia is typically in early adulthood [3], and after that schizophrenia is often associated with recurrent hospitalizations, poor social functioning, low education, and high-unemployment rates. The societal burden of schizophrenia is substantial [4-6]; it has been estimated that the total cost of schizophrenia in the USA at 2013 was around $\$ 150$ billion [7], and in England, it was around $£ 20$ billion in 2010/2011 [8]. From these costs, indirect costs attributed to unemployment have been shown to be largest components of the total economic burden of schizophrenia [5], indicating that schizophrenia can lead to occupational and social exclusion [9].

However, it remains unclear what are the prospects of individuals with schizophrenia in terms of occupational (i.e., employment, income, and education) and social inclusion (i.e., cohabitation) over the work life course. Considerable variation in the employment rate of individuals with schizophrenia, estimating ranging from 10 to $30 \%$ [10-13], has been documented, and schizophrenia has also been associated with lower income among those who are able to work [14], and also to lower likelihood of obtaining secondary or higher education [15]. Moreover, schizophrenia has been associated with lower rates of marriage and higher rates of separation and divorce $[16,17]$, indicating that many individuals with schizophrenia are likely to end up living alone. This-in combination with unemployment and low education-is likely to lead to overall occupational and social exclusion, but there is lack of empirical data that would cover the whole work life course on the topic. In addition, to our knowledge, previous studies have not examined whether these associations are different for those individuals who have less chronic course of the disease, i.e., they are not recurrently hospitalized. Although only around $14 \%$ of individuals with schizophrenia will achieve both clinical and functional recovery [18], supported employment has been shown to be effective at placing individuals with schizophrenia in competitive employment across the world $[19,20]$.

To examine both occupational and social exclusion over the work life course, our main was to examine how schizophrenia with an onset before the age of 25 affects later employment status, income, educational attainment, and cohabitant status using Danish population longitudinal cohort register data, which enables us to link national registers and to undertake a comprehensive and nationwide analysis. We decided to focus on those individuals who had the first onset of schizophrenia in early adulthood as it is the time period when most young adults complete their educational degrees and start their careers. Our secondary aim was to examine whether these associations differed among those participants who potentially had less chronic course of schizophrenia, i.e., they were diagnosed with schizophrenia before the age of 25 , but did not receive treatment in a psychiatric inpatient or outpatient clinic for schizophrenia after the age of 25 . Finally, we examined whether family socioeconomic and psychiatric history, which are established risk factors of schizophrenia [21, 22], influenced these associations. We reported both odds ratios and absolute proportions; whereas odds ratios are important for exploring risks, absolute proportions are more informative for service planning and general public.

\section{Materials and methods}

\section{Study population}

Since 1968, all residents in Denmark have a unique personal identification number allowing data to be linked at an individual level across administrative population registers. We used the Danish Civil Registration System which includes demographic details and links to parents as well as continuous updates on place of residence and vital status [23]. Our study population included all individuals born in Denmark between January 1, 1955 and December 31, 1991, who were alive at their 25th birthday. The study was approved by the Danish Data Protection Agency and did not require ethical approval according to Danish laws.

\section{Schizophrenia assessment}

Study population and their parents were linked to the Danish Psychiatric Central Research Register [24], which covers all psychiatric inpatient admissions starting from 1969 and from 1995 all outpatient and emergency unit visits. Individuals were defined as having schizophrenia (ICD-10 code F20; ICD-8 codes 295.x9 [excluding 295.79 (schizoaffective disorder)]) if they had been admitted to a psychiatric hospital, had received outpatient psychiatric care, or had visited psychiatric emergency unit. Studies have shown that diagnosis of schizophrenia has high validity in the Danish Psychiatric Central Register [25]. Date of onset was defined as the first day that an individual was given a diagnosis of schizophrenia. For the study population, first date of schizophrenia diagnosis between age 15 and 25 and the first date of schizophrenia diagnosis after age 25 were defined.

\section{Labour market and social exclusion outcomes}

Employment status was derived from the Employment Classification Module (AKM) [26], where based on the main source of income or employment, annual status of most important employment is classified. Individuals who were unemployed, retired (including early retirement and disability pension), and otherwise not working were categorized as outside the labour market. Annual wage or selfemployment earnings were acquired from the Integrated 
Database for Labour Market Research (IDA) [26] and measured from all individuals who had permanent residence in Denmark at the 31th of December. Earnings were deflated using the consumer price index (base year 2015). Highest completed education was acquired from the Danish education registries [27], which is updated annually. Individuals with only primary school education were categorized as having no secondary education. In addition to these labour market outcomes, current cohabitant status ( $1=$ living alone; $0=$ not living alone $)$ was used as an outcome for social exclusion.

\section{Covariates}

Information of parental employment, income, and highest completed education was obtained when the participants were 25 years old. The same definitions (as described above) were used and for both father and mother. In addition, and in line with previous research, low income was defined as less than annual earnings of 30,000 DKK (\$4500) [28], which were deflated using the consumer price index, base year 2015. For both parents, parental socioeconomic score was calculated where one point was given to unemployment, low income, and no secondary education. This resulted in a score ranging from 0 to 3 . History of parental mental disorders was obtained from the Danish Psychiatric Central Register [24], and parental mental disorders were categorized into two groups $(0=$ no

Fig. 1 On the left, odds ratios for the association between schizophrenia diagnosis between ages 15 and 25 with being not employed from 25 to 61 years. On the middle, the association between schizophrenia with being not employed from 25 to 61 years for a subgroup of individuals who were not rehospitalized for schizophrenia after the age of 25. On the right, percentages of individuals not being employed mental disorders; $1=$ any mental disorder) according to the criteria presented in earlier study [3].

\section{Statistical analyses}

The association between incidence of schizophrenia with (1) not being employed, (2) having no secondary or higher education after primary school, and (3) not cohabiting or being married were examined using a series of logistic regression analyses where diagnosis of schizophrenia between age 15 and 25 (yes/no) was the exposure, and status of employment, education, and cohabitation between age 25 and 61 were used as outcomes. In these analyses, individuals who were not diagnosed with schizophrenia between the age 15 and 25 were used as comparison group. These same associations were additionally examined in a subgroup of individuals with schizophrenia who were not rehospitalized for schizophrenia after the age of 25 -i.e., they did not receive treatment in a psychiatric inpatient or outpatient clinic for schizophrenia after the age of 25 . In these analyses, all those individuals who were not diagnosed with schizophrenia before the age when the outcome was measured were used as comparison group, and thus, individuals who were diagnosed with schizophrenia for the first time, before the age when the outcome was measured, were excluded. All analyses were adjusted for sex and the year of birth. Results of these analyses were reported at every year after the age of 25 in Figs. 1, 3 and 4. Annual mean levels of income are reported in Fig. 2. Additional analyses were conducted where associations were adjusted for parental history of

\section{Not employed}

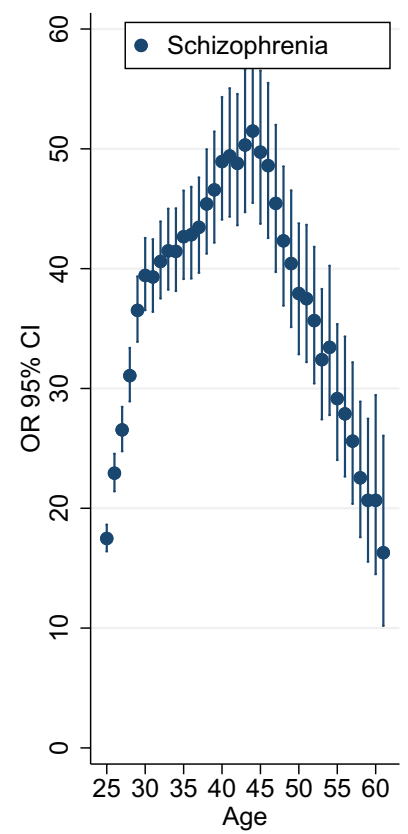

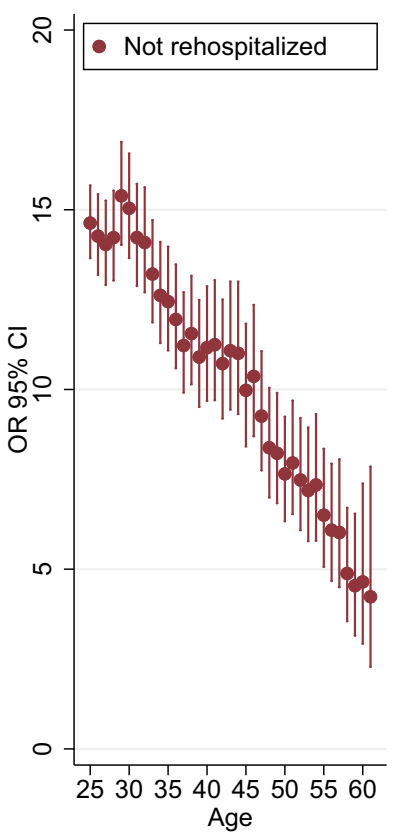

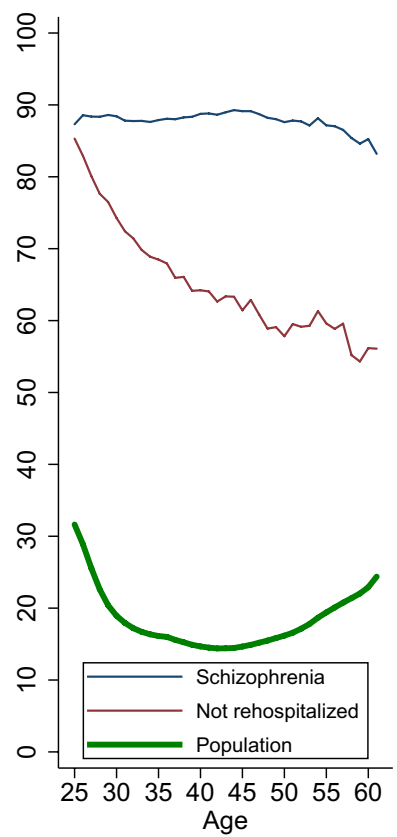




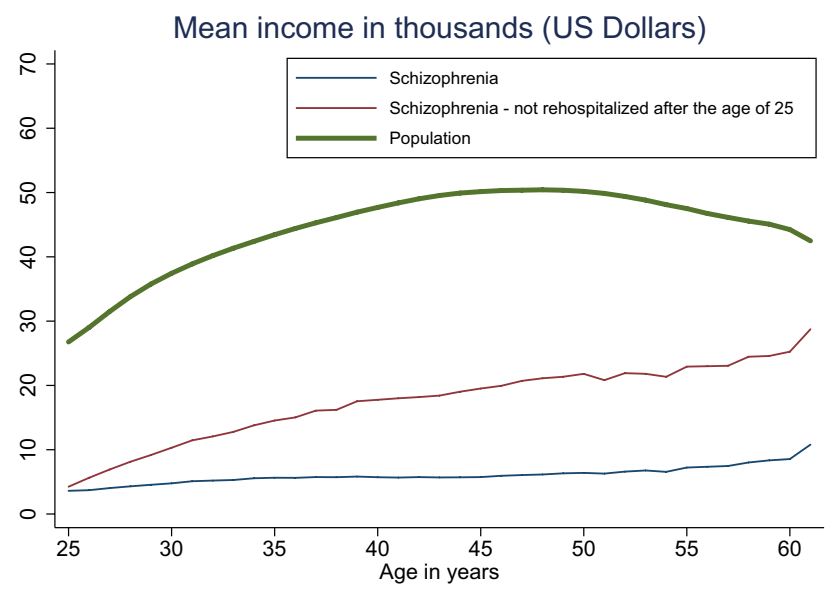

Fig. 2 Mean income for individuals (a) with schizophrenia before the age of 25 , (b) with schizophrenia before the age of 25 who had not been rehospitalized for schizophrenia after the age of 25 , and (c) without schizophrenia before the age of 25

mental disorders and parental socioeconomic status. These results are reported for at ages 30, 40, and 50 in Table 1. Stata 15.1 was used for all analyses.

\section{Results}

\section{Sample}

In total, 2,390,127 individuals (48.8\% women) were included. Between ages 15 and 25, 9448 individuals were diagnosed with schizophrenia and 20,633 participants were diagnosed with schizophrenia after the age of 25. Out of the 9448 individuals who were diagnosed with schizophrenia before the age of 25, a total of 6328 (69\%) were rehospitalized with schizophrenia after the 25th birthday. Descriptive statistics, number of participants, and labour market outcomes, for the three different groups (schizophrenia, schizophrenia not rehospitalized after the age of 25 , and population) are reported in Online Appendix Tables 1 and 2 , respectively. At the age 30, from the individuals who were not employed, $8.9 \%$ did not also have any secondary or higher education and $9.5 \%$ were not cohabiting. Contrary to this, from the individuals who were diagnosed with schizophrenia before the age of 25 and who were not employed at the age of $30,64.4 \%$ did not also have any secondary or higher education and $74.4 \%$ were not cohabiting.

\section{Employment}

Figure 1 shows (a) the associations between schizophrenia with not being employed, (b) the associations between schizophrenia with not being employed and for individuals who were not hospitalized for schizophrenia after the age of 25 and (c) the absolute proportions of not being employed for individuals with schizophrenia, subgroup of schizophrenia without rehospitalization after the age of 25 , and population without schizophrenia before the age of 25 . Over the whole follow-up, schizophrenia was associated with higher likelihood of not being employed. These odds ratios increased from 17.5 (95\% CI 16.4-18.6) to 48.9 (95\% CI 44.1-54.3) between ages 25 and 45 , and then decreased (at the age of 60: odds ratio [OR] 20.7, 95\% CI 14.5-29.5) for individuals diagnosed with schizophrenia. For the subgroup of schizophrenia without rehospitalization after the age of 25 , patterns were differed compared to the primary analysis; the odds ratios for not being employed were 14.6 (95\% CI 13.7-15.7) at the age of 25 . These odds ratios were then first remained stable (at the age of 30: OR 15.0, 95\% CI 13.7-16.6), and then steadily decreased to 4.6 (95\% CI 2.9-7.4) at the age of 60 . In absolute proportions, at the age of $25,87 \%$ of the individuals with schizophrenia were not employed, and at the age of $60,85 \%$ of the individuals with schizophrenia were not employed. Contrary to these findings, whereas $85 \%$ of the individuals with schizophrenia who were not rehospitalized after the age of 25 were not being employed at the age of $25,56 \%$ were not being employed at the age of 60 .

\section{Income levels}

Figure 2 shows the mean income levels for individuals with schizophrenia, subgroup of individuals with schizophrenia who have not been rehospitalized after the age of 25, and individuals who were not diagnosed with schizophrenia before the age of 25. Between ages 25 and 61, individuals with schizophrenia have cumulative earnings around $\$ 224,000$ (1,504,000 DKK), which is $13.6 \%$ of the amount that the individuals who have not been diagnosed with schizophrenia earn ( $\$ 1,643,000$ corresponding to 11.05 million DKK). For those individuals who have not been rehospitalized for schizophrenia after the age of 25 , the corresponding sum was around three times more $(\$ 648,000$ corresponding to 4.35 million DKK), which is $39.4 \%$ of the amount that the individuals who have not been diagnosed with schizophrenia earn.

\section{Cohabitation status}

Figure 3 shows the associations: (a) the overall associations between schizophrenia with not cohabiting, (b) the associations between schizophrenia with not cohabiting among those individuals who were not hospitalized for schizophrenia after the age of 25 , and (c) the absolute proportions of individuals for three groups (schizophrenia, subgroup of schizophrenia without rehospitalization after the age of 25 , 
Fig. 3 On the left, the association between schizophrenia with not cohabiting. On the middle, the associations between schizophrenia with not cohabiting for a subgroup of individuals who were not rehospitalized for schizophrenia after the age of 25 . On the right, percentages of individuals not cohabiting in three groups (schizophrenia, schizophrenia-not rehospitalized subgroup, population)

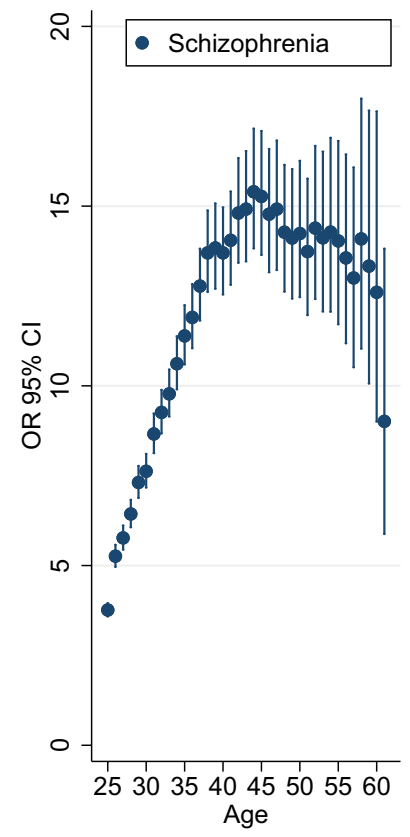

Not cohabiting
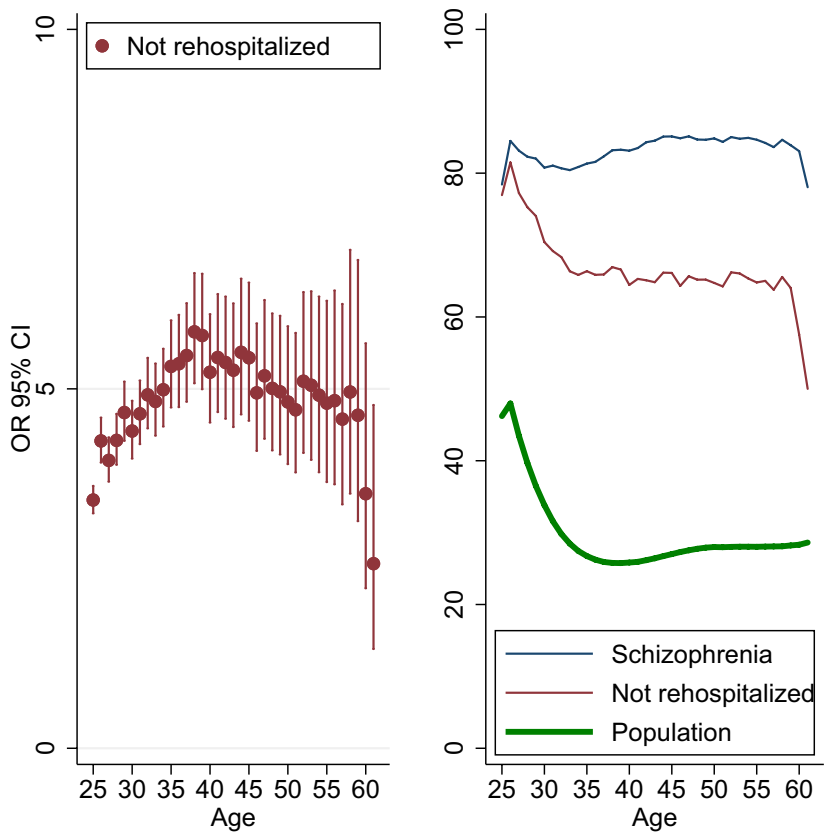

Fig. 4 On the left, the associations between schizophrenia with having no secondary or higher education. On the middle, the associations between schizophrenia with having no secondary or higher education for a subgroup of individuals who were not hospitalized for schizophrenia after the age of 25 . On the right, percentsecondary or higher education in three groups (schizophrenia, schizophrenia-not rehospitalized subgroup, population) ages of individuals having no

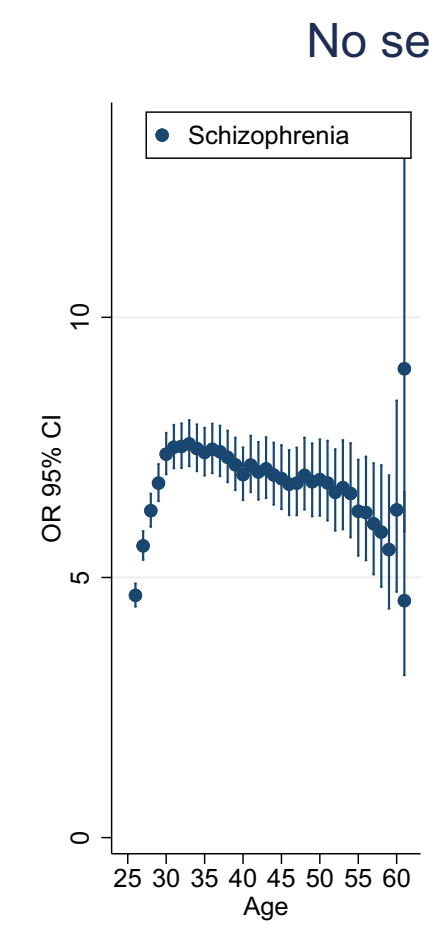

and population who were not diagnosed with schizophrenia before the age of 25). Schizophrenia was associated with higher odds of not cohabiting - and these odds increased over three times between the ages of 25-40: at the age of 25, odds were 3.8 (95\% CI 3.6-4.0), and at the age of 40, they were 13.7 (95\% CI 12.5-15.0). Although these associations were present also for those individuals with schizophrenia
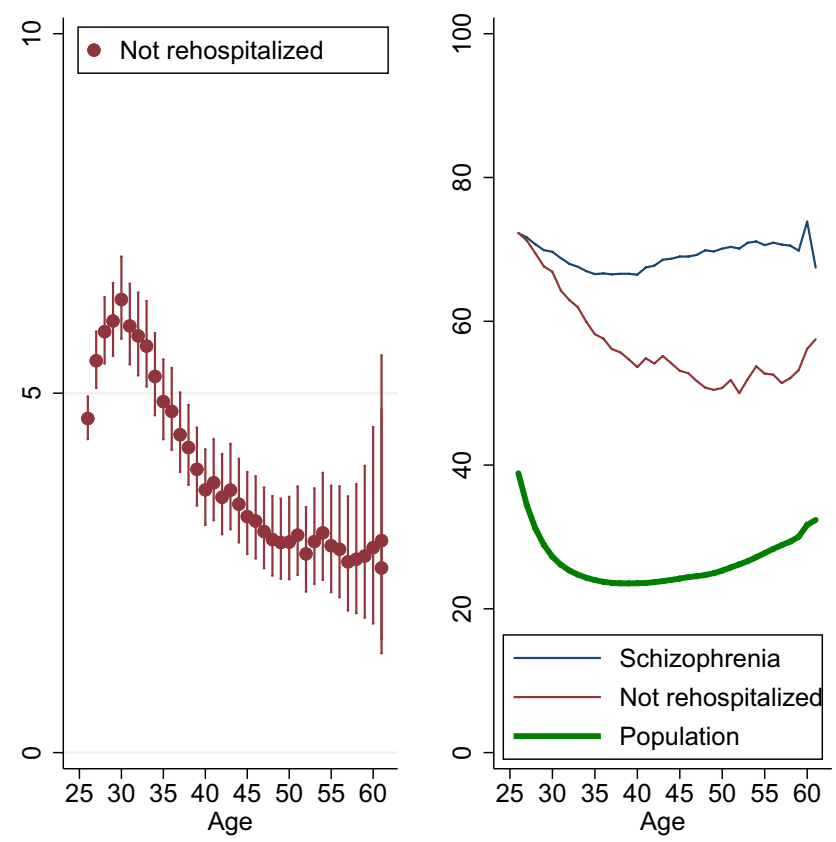

who were not rehospitalized after the age of 25 [at the age of 25 , OR 3.5 (95\% CI 3.3-3.6); at the age of 40, OR 5.2 (95\% CI 4.5-6.0)], the increase was more modest. In absolute proportions, whereas $46 \%$ of the population was not cohabiting at the age of $25,78 \%$ of the individuals with were not cohabiting. At the age of $40,26 \%$ of the population was not cohabiting, whereas $83 \%$ of the individuals with 
schizophrenia and $64 \%$ of the individuals with schizophrenia who were not rehospitalized were not cohabiting.

\section{Completing secondary or higher education}

Figure 4 shows the associations (a) between schizophrenia with not having any secondary or higher education and (b) between schizophrenia with not having any secondary or higher education among those individuals who were not hospitalized for schizophrenia after the age of 25 . Individuals with schizophrenia had increased odds ratios of not completing secondary or higher education (at the age of 25: OR 4.7, 95\% CI 4.4-4.9; at the age of 35: OR 7.4, 95\% CI 7.0-7.9). These odds ratios showed little decrease over time (at the age of 55: OR 6.3, 95\% CI 5.4-7.3). For those individuals who were not rehospitalized after the age of 25 , patterns were similar (at the age of 25: OR 4.7, 95\% CI 4.4-4.9) except that the decrease in odds ratios was more rapid [at the age of 40 , the odds ratio was 3.6 (95\% CI 3.2-4.2)].

\section{Sensitivity analyses}

The schizophrenia-labour market associations adjusted for parental socioeconomic measures and parental history of mental disorders are shown in Table 1. In general, adjustment for parental socioeconomic measures (model 2 and model 3) had very little, if any, effect on the associations between schizophrenia with labour market outcomes. Additional adjustment for parental history of mental disorders diluted the associations also very little-for example, odds ratio for being unemployed was $6 \%$ lower when parental socioeconomic measures and history of mental disorders were taken into account. Similar patterns were found when associations between schizophrenia, which had not been rehospitalized with schizophrenia diagnosed, with labour market outcomes were examined (please see, Online Appendix Table 3).

\section{Discussion}

\section{Summary of the main results}

Using Danish longitudinal population data, we examined what are the prospects of individuals diagnosed with schizophrenia in terms of employment, income, educational attainment, and cohabitation status over the whole work life course. Our main findings suggest that schizophrenia diagnosis between age 15 and 25 is associated with over 20 times higher risk of not being employed, over six times higher risk of having no secondary or higher education, and over ten times higher risk of living alone. These associations were, however, two-to-three times lower for a subgroup of individuals who had potentially a less chronic course of schizophrenia, i.e., they did not receive treatment in a psychiatric inpatient or outpatient clinic for schizophrenia after the age of 25. Whereas the work life course salary earnings of individuals with schizophrenia were around $\$ 224,000$,
Table 1 Associations between schizophrenia diagnosis between ages 15 and 25 with labour market outcomes (employment, income, and education) and status of cohabitation at the age of 30 , 40 , and 50

\begin{tabular}{|c|c|c|c|c|c|c|}
\hline & \multicolumn{2}{|c|}{ Not employed } & \multicolumn{2}{|c|}{ Not cohabiting } & \multicolumn{2}{|c|}{ Low education } \\
\hline & OR & $95 \% \mathrm{CI}$ & OR & $95 \% \mathrm{CI}$ & OR & $95 \% \mathrm{CI}$ \\
\hline \multicolumn{7}{|c|}{ Age $30(n=1,606,691)$} \\
\hline Model 1 & 38.24 & $34.93-41.87$ & 7.11 & $6.62-7.64$ & 7.72 & $7.25-8.22$ \\
\hline Model 2 & 37.71 & $34.44-41.30$ & 7.03 & $6.55-7.55$ & 7.62 & $7.15-8.12$ \\
\hline Model 3 & 37.37 & $34.12-40.93$ & 6.98 & $6.50-7.50$ & 7.59 & $7.12-8.09$ \\
\hline Model 4 & 35.83 & $32.71-39.25$ & 6.78 & $6.32-7.29$ & 7.25 & $6.80-7.74$ \\
\hline \multicolumn{7}{|c|}{ Age $40(n=1,142,861)$} \\
\hline Model 1 & 50.32 & $44.38-57.05$ & 13.42 & $12.05-14.93$ & 7.43 & $6.81-8.11$ \\
\hline Model 2 & 50.92 & $44.90-57.75$ & 13.34 & $11.98-14.84$ & 7.61 & $6.96-8.31$ \\
\hline Model 3 & 51.56 & $45.45-58.49$ & 13.30 & $11.95-14.80$ & 7.78 & $7.12-8.50$ \\
\hline Model 4 & 49.26 & $43.41-55.90$ & 12.78 & $11.48-14.23$ & 7.51 & $6.87-8.21$ \\
\hline \multicolumn{7}{|c|}{ Age $50(n=565,007)$} \\
\hline Model 1 & 40.37 & $33.43-48.75$ & 14.55 & $12.21-17.34$ & 7.42 & $6.47-8.52$ \\
\hline Model 2 & 40.74 & $33.73-49.22$ & 14.45 & $12.13-17.22$ & 7.68 & $6.68-8.83$ \\
\hline Model 3 & 41.30 & $34.18-49.91$ & 14.42 & $12.10-17.18$ & 7.86 & $6.83-9.04$ \\
\hline Model 4 & 39.69 & $32.83-47.98$ & 13.94 & $11.70-16.62$ & 7.69 & $6.68-8.85$ \\
\hline
\end{tabular}

Model 1: sex and birth year adjusted

Model 2: Model $1+$ father's SES score

Model 3: Model 2+ mother's SES score

Model 4: Model 3+ parental history of mental disorders 
similar figure for a subgroup of individuals who had not been rehospitalized for schizophrenia was around $\$ 648,000$, which are around $14 \%$ and $39 \%$, respectively, of the amount the individuals who have not been diagnosed with schizophrenia before the age of 25 earn. Taken together, these findings suggest that although schizophrenia is associated with high risk of occupational exclusion and living alone over the work life course, individuals with potentially less chronic course of schizophrenia are able to participate in the working life at least to some degree. Best of our knowledge, this is the first population-based study that has investigated the associations of schizophrenia with employment, income, and status of cohabitation from a work life course perspective.

\section{Comparison with the previous studies}

Employment is often stated as a recovery goal in schizophrenia. Previous studies have shown that the unemployment rate for individuals with schizophrenia is very high, from 80 to $90 \%$ [4, 10, 11], and that individuals with schizophrenia have a high-unemployment rate and low earnings before the onset of disease [29, 30], suggesting that ability to work is impaired already during the prodromal phase. Here, the unemployment was on average around $88 \%$. For those individuals who did not receive treatment in a psychiatric inpatient or outpatient clinic for schizophrenia after the age of 25 , and thus presumably had a less chronic course, the unemployment rate steadily decreased from around $75 \%$ to around 55\%. These novels findings indicate that a less chronic course of schizophrenia is associated with improving rate of employment over the work life course. Best of our knowledge, previous studies have not examined whether there is a subgroup of individuals with schizophrenia who do better than others in terms of employment and educationrelated outcomes.

\section{Plausible mechanisms}

There are some plausible explanations for the current findings. It is well known that cognitive deficits, which are present before the onset of psychotic symptoms [31], are a core feature of schizophrenia [32, 33], and a likely explanation why schizophrenia is from the early on associated with unemployed. More importantly, deficits in social functioning could also explain why schizophrenia is associated with unemployment. However, it is also well documented that individuals with schizophrenia can be exposed to discrimination in many areas of life [34], which is likely to lead to a loss of job and difficulties in findings new employment. Low parental socioeconomic position and history of parental mental disorders are both established risk factors for developing schizophrenia [21, 22]. When adjusted for these factors, the associations between schizophrenia and labour market outcomes were only slightly attenuated. This indicates that although these factors contribute to the development of schizophrenia, they made little-if any-difference whether individuals with schizophrenia were able to work or to get an education in the present study. Finally, although a longer duration of untreated psychosis is associated with more severe positive and negative symptoms, it has not been associated with employment outcomes [35]. Whether a shorter duration of untreated psychosis would be associated with positive education-related outcomes remains unknown.

\section{Strengths and limitations}

Main strengths of the current study are Danish national registers that provided rich information for the entire population. Individual-level labour market [26] and education registries [27] have high coverage and reliability. Danish psychiatric hospitals and outpatient facilities are public, and all admissions and discharges are recorded in the registers. All treatment is also free of charge. Thus, the current study does not suffer from selective non-response, which likely biases survey studies conducted on the topic.

When interpreting current findings, the number of limitations needs to be taken into account. First, selective attrition due to higher mortality in individuals with schizophrenia could partly explain some of the current findings; we did not have data on the severity of schizophrenia, and as schizophrenia is a very heterogeneous mental disorder with high comorbidity with other mental disorders, it is likely that those individuals with worse symptoms are more likely to die. This naturally creates a survival effect where more healthy individuals are likely to survive longer. This survival effect could especially partly explain why individuals with schizophrenia seem to get better over time. Second, although the ICD-10 schizophrenia diagnosis in the Danish Psychiatric Central Register has high diagnostic validity, it has been showed that a small percentage of cases in the Danish Psychiatric Central Register have been mistakenly registered as having schizophrenia when another psychiatric diagnosis was given [25]. In the ICD-8, schizophrenia diagnosis has been used to more severe cases with a longer duration [36]. In addition, it is also likely that there are some individuals with schizophrenia who have not been diagnosed or has been misdiagnosed. These issues could introduce bias one way or to another to our findings. Third, a large fraction of individuals with schizophrenia are first diagnosed after their 25th birthday, and it is not known whether their labour market prospects are less dire than those diagnosed before their 25th birthday. Thus, current study results should be treated as an estimate how schizophrenia with an onset before the age of 25 is related to labour market outcomes over the long run. Fourth, in Denmark, there has been numerous societal changes related to 
labour market and education from 1980 to 2015, which could affect current results and, therefore, possible cohort effects could in part explain current study findings. All individuals included in the study were born in Denmark, and thus, present findings might not be generalizable to individuals who were born elsewhere. Fifth, although current cohabitation status is often used as a measure of structural social support and social exclusion $[37,38]$, it is a very crude measure that does not capture quality and complexity of social relationships. Finally, $98 \%$ of the population in Denmark is assigned to a specific general practitioner (GP) who provides taxfunded public healthcare services. GP provides medical care and also acts as a gatekeeper to secondary health being the first contact in most chronic diseases, including schizophrenia. As our follow-up time for younger cohorts is relatively short and our data include outpatient and emergency unit visits starting from 1995, it is possible that our estimate of rehospitalization rate does not reflect the actual rehospitalization rate for individuals with schizophrenia. These issues could also introduce bias to our findings.

\section{Conclusions}

To conclude, individuals with schizophrenia are at high risk of being outside the labour market and living alone throughout their entire life, resulting in an enormous societal loss in earnings. Although these associations are relatively unaffected by parental socioeconomic status and psychiatric history, individuals with schizophrenia who had potentially a less chronic course experienced a gradual but substantial improvement throughout their work life.

Acnowledgements Open access funding provided by University of Helsinki including Helsinki University Central Hospital.

Funding This work was supported by the Academy of Finland (310591 to $\mathrm{CH}$ ), the Stanley Medical Research Institute (PBM, AT, EA, CBP), and Lundbeck Foundation, Denmark (PBM, AT, EA, CBP). JJM received a John Cade Fellowship APP1056929 from the National Health and Medical Research Council and a Niels Bohr Professorship from the Danish National Research Foundation.

\section{Compliance with ethical standards}

Conflict of interest None declared.

Ethics statement The study was approved by the Danish Data Protection Agency and did not require ethical approval according to Danish laws.

Open Access This article is distributed under the terms of the Creative Commons Attribution 4.0 International License (http://creativeco mmons.org/licenses/by/4.0/), which permits unrestricted use, distribution, and reproduction in any medium, provided you give appropriate credit to the original author(s) and the source, provide a link to the Creative Commons license, and indicate if changes were made.

\section{References}

1. Laursen TM, Nordentoft M, Mortensen PB (2014) Excess early mortality in schizophrenia. Annu Rev Clin Psychol 10:425-448. https://doi.org/10.1146/annurev-clinpsy-032813-153657

2. Charlson FJ, Ferrari AJ, Santomauro DF et al (2018) Global epidemiology and burden of schizophrenia: findings from the global burden of disease study 2016. Schizophr Bull. https://doi. org/10.1093/schbul/sby058

3. Pedersen CB, Mors O, Bertelsen A et al (2014) A comprehensive nationwide study of the incidence rate and lifetime risk for treated mental disorders. JAMA Psychiatry 71:573-581. https:// doi.org/10.1001/jamapsychiatry.2014.16

4. Evensen S, Wisløff T, Lystad JU et al (2016) Prevalence, employment rate, and cost of schizophrenia in a high-income welfare society: a population-based study using comprehensive health and welfare registers. Schizophr Bull 42:476-483. https://doi. org/10.1093/schbul/sbv141

5. Chong HY, Teoh SL, Wu DB-C et al (2016) Global economic burden of schizophrenia: a systematic review. Neuropsychiatr Dis Treat 12:357-373. https://doi.org/10.2147/NDT.S96649

6. Jin H, Mosweu I (2017) The societal cost of schizophrenia: a systematic review. Pharmacoeconomics 35:25-42. https://doi. org/10.1007/s40273-016-0444-6

7. Cloutier M, Sanon Aigbogun M, Guerin A et al (2016) The economic burden of schizophrenia in the United States in 2013. J Clin Psychiatry 2012:764-771. https://doi.org/10.4088/JCP.15m10278

8. Commission Schizophrenia (2012) The abandoned illness: a report from the Schizophrenia Commission. The Schizophrenia Commission, London

9. Leff J, Wagner R (2006) Social inclusion of people with mental illness. Cambridge University Press, Cambridge

10. Marwaha S, Johnson S, Bebbington P et al (2007) Rates and correlates of employment in people with schizophrenia in the UK, France and Germany. Br J Psychiatry 191:30-37. https://doi. org/10.1192/bjp.bp.105.020982

11. Marwaha S, Johnson S (2004) Schizophrenia and employment: a review. Soc Psychiatry Psychiatr Epidemiol 39:337-349. https:// doi.org/10.1007/s00127-004-0762-4

12. Zivin K, Bohnert ASB, Mezuk B et al (2011) Employment status of patients in the VA health system: implications for mental health services. Psychiatr Serv 62:35-38. https://doi.org/10.1176/ ps.62.1.pss6201_0035

13. Byrne M, Agerbo E, Eaton WW, Mortensen PB (2004) Parental socio-economic status and risk of first admission with schizophrenia - a Danish national register based study. Soc Psychiatry Psychiatr Epidemiol 39:87-96. https://doi.org/10.1007/s0012 7-004-0715-y

14. Kessler RC, Heeringa S, Lakoma $M$ et al (2008) Individual and societal effects of mental disorders on earnings in the United States: results from the National Comorbidity Survey Replication. Am J Psychiatry 165:703-711

15. Tempelaar WM, Termorshuizen F, MacCabe JH et al (2017) Educational achievement in psychiatric patients and their siblings: a register-based study in 30000 individuals in the Netherlands. Psychol Med 47:776-784. https://doi.org/10.1017/S003329171 6002877

16. Cohen A, Patel V, Thara R, Gureje O (2008) Questioning an axiom: better prognosis for schizophrenia in the developing world? Schizophr Bull 34:229-244. https://doi.org/10.1093/schbu $1 /$ sbm 105 
17. Salokangas RKR, Honkonen T, Stengård E, Koivisto AM (2001) To be or not to be married - that is the question of quality of life in men with schizophrenia. Soc Psychiatry Psychiatr Epidemiol 36:381-390. https://doi.org/10.1007/s001270170028

18. Jääskeläinen E, Juola P, Hirvonen N et al (2013) A systematic review and meta-analysis of recovery in schizophrenia. Schizophr Bull 39:1296-1306. https://doi.org/10.1093/schbul/sbs130

19. Metcalfe JD, Drake RE, Bond GR (2018) Economic, labor, and regulatory moderators of the effect of individual placement and support among people with severe mental illness: a systematic review and meta-analysis. Schizophr Bull 44:22-31. https://doi. org/10.1093/schbul/sbx132

20. Bond GR, Drake RE, Becker DR (2012) Generalizability of the individual placement and support (IPS) model of supported employment outside the US. World Psychiatry 11:32-39. https:// doi.org/10.1016/j.wpsyc.2012.01.005

21. Agerbo E, Sullivan PF, Vilhjálmsson BJ et al (2015) Polygenic risk score, parental socioeconomic status, family history of psychiatric disorders, and the risk for schizophrenia: a Danish population-based study and meta-analysis. JAMA Psychiatry 72:635-641. https://doi.org/10.1001/jamapsychiatry.2015.0346

22. Dean K, Stevens H, Mortensen PB et al (2010) Full spectrum of psychiatric outcomes among offspring with parental history of mental disorder. Arch Gen Psychiatry 67:822-829. https://doi. org/10.1001/archgenpsychiatry.2010.86

23. Pedersen CB (2011) The Danish civil registration system. Scand J Public Health 39:22-25. https://doi.org/10.1177/1403494810 387965

24. Mors O, Perto GP, Mortensen PB (2011) The Danish psychiatric central research register. Scand J Public Health 39:54-57. https:// doi.org/10.1177/1403494810395825

25. Uggerby P, Østergaard SD, Røge R et al (2013) The validity of the schizophrenia diagnosis in the Danish Psychiatric Central Research Register is good. Dan Med J 60:A4578

26. Petersson F, Baadsgaard M, Thygesen LC (2011) Danish registers on personal labour market affiliation. Scand J Public Health 39:95-98. https://doi.org/10.1177/1403494811408483

27. Jensen VM, Rasmussen AW (2011) Danish education registers. Scand J Public Health 39:91-94. https://doi.org/10.1177/14034 94810394715

28. Westergaard-Nielsen N, Agerbo E, Eriksson T, Mortensen PB (2004) Mental illness: gender differences with respect to marital status and labour market outcomes. In: Marcotte DE, Wilcox-Gök
V (eds) The economics of gender and mental illness. Elsevier, Amsterdam, pp 73-94

29. Agerbo EMS, Byrne MMS, Eaton WWP, Mortensen PBM (2004) Marital and labor market status in the long run in schizophrenia. Arch Gen Psychiatry 61:28-33. https://doi.org/10.1001/archp syc.61.1.28

30. Davidson M, Kapara O, Goldberg S et al (2015) A Nation-wide study on the percentage of schizophrenia and bipolar disorder patients who earn minimum wage or above. Schizophr Bull 42:15. https://doi.org/10.1093/schbul/sbv023

31. Dickson H, Laurens KR, Cullen AE, Hodgins S (2012) Metaanalyses of cognitive and motor function in youth aged 16 years and younger who subsequently develop schizophrenia. Psychol Med 42:743-755. https://doi.org/10.1017/S0033291711001693

32. Kahn RS, Keefe RSE (2013) Schizophrenia is a cognitive illness: time for a change in focus. JAMA Psychiatry 70:1107-1112. https ://doi.org/10.1001/jamapsychiatry.2013.155

33. Schaefer J, Giangrande E, Weinberger DR, Dickinson D (2013) The global cognitive impairment in schizophrenia: consistent over decades and around the world. Schizophr Res 150:42-50. https:// doi.org/10.1016/j.schres.2013.07.009

34. Thornicroft G, Brohan E, Rose D et al (2009) Global pattern of experienced and anticipated discrimination against people with schizophrenia: a cross-sectional survey. Lancet 373:408-415. https://doi.org/10.1016/S0140-6736(08)61817-6

35. Penttilä M, Jaäskelainen E, Hirvonen N et al (2014) Duration of untreated psychosis as predictor of long-term outcome in schizophrenia: systematic review and meta-analysis. Br J Psychiatry 205:88-94. https://doi.org/10.1192/bjp.bp.113.127753

36. Munk-Jørgensen P (1985) The schizophrenia diagnosis in Denmark. Acta Psychiatr Scand 72:266-273. https://doi. org/10.1111/j.1600-0447.1985.tb02605.x

37. Hakulinen C, Pulkki-Råback L, Jokela M et al (2016) Structural and functional aspects of social support as predictors of mental and physical health trajectories: whitehall II cohort study. J Epidemiol Community Health 70:710-715. https://doi.org/10.1136/ jech-2015-206165

38. Sacker A, Ross A, MacLeod CA et al (2017) Health and social exclusion in older age: evidence from Understanding Society, the UK household longitudinal study. J Epidemiol Community Health 71:681-690. https://doi.org/10.1136/jech-2016-208037 